\title{
EVOLUTIONARY TIMESCALE OF THE PULSATING WHITE DWARF G117-B15A: THE MOST STABLE OPTICAL CLOCK KNOWN
}

\author{
S. O. KEPLER \\ Instituto de Física da UFRGS, 91501-900 Porto Alegre, RS Brazil; kepler@if.ufrgs.br \\ Anjum Mukadam, D. E. Winget, R. E. Nather, and T. S. Metcalfe \\ Department of Astronomy, University of Texas, Austin, TX 78712 \\ M. D. Reed and S. D. Kawaler \\ Department of Physics and Astronomy, Iowa State University, Ames, Iowa 50011 \\ AND \\ Paul A. Bradley \\ Los Alamos National Laboratory, Los Alamos, NM 87545 \\ Received 2000 January 31; accepted 2000 March 30; published 2000 May 4
}

\begin{abstract}
We observe G117-B15A, the most precise optical clock known, to measure the rate of change of the main pulsation period of this blue-edge DAV white dwarf. Even though the obtained value is only within $1 \sigma, \dot{P}=$ $(2.3 \pm 1.4) \times 10^{-15} \mathrm{~s} \mathrm{~s}^{-1}$, it is already constraining the evolutionary timescale of this cooling white dwarf star. Subject headings: stars: evolution — stars: individual (G117-B15A) — stars: oscillations
\end{abstract}

\section{INTRODUCTION}

We report our continuing study of the star G117-B15A (also called RY LMi and WD 0921+354), one of the hottest of the pulsating white dwarfs with hydrogen atmospheres, the DAV or ZZ Ceti stars (McGraw 1979). McGraw \& Robinson (1976) found that the star was variable, and Kepler et al. (1982) studied its light curve and found six pulsation modes. The dominant mode is at $215 \mathrm{~s}$, has a fractional amplitude of 22 millimodulation amplitudes, and is stable in amplitude and phase. The other, smaller pulsation modes vary in amplitude from night to night (Kepler et al. 1995). Because the DAVs appear to be normal stars except for their variability (Robinson 1979; Bergeron et al. 1995), it is likely that the DAV structural properties are representative of all DA white dwarfs.

The rate of change of a pulsation period is directly related to the evolutionary timescale of a white dwarf, allowing us to directly infer the age of a white dwarf since its formation. We have been working since 1975 to measure the rate of period change with time $(\equiv \dot{P})$ for the $P=215$ s periodicity of $\mathrm{G} 117$ B15A, and the Kepler et al. (1991) determination was $\dot{P}=$ $(12.0 \pm 3.5) \times 10^{-15} \mathrm{~s} \mathrm{~s}^{-1}$, including all data obtained from 1975 through 1990.

Kepler (1984) demonstrated that the observed variations in the light curve of G117-B15A are due to nonradial $g$-mode pulsations, and therefore the timescale for period change is directly proportional to the cooling timescale.

For comparison, the most stable atomic clocks have rates of period change of the order of $\dot{P} \simeq 2 \times 10^{-14} \mathrm{~s} \mathrm{~s}^{-1}$, while the most precise millisecond pulsars have $\dot{P} \simeq 10^{-20} \mathrm{~s} \mathrm{~s}^{-1}$ (Kaspi, Taylor, \& Ryba 1994 and references therein). Since the stability of a clock is measured by $P / \dot{P}, \mathrm{G} 117-\mathrm{B} 15 \mathrm{~A}$ has the same order of stability as the most stable millisecond pulsar.

G117-B15A is the first pulsating white dwarf to have its main pulsation mode index identified. The $215 \mathrm{~s}$ mode is an $l=1$, as determined by comparing the ultraviolet pulsation amplitude (measured with the Hubble Space Telescope) to the optical amplitude (Robinson et al. 1995). Robinson et al. (1995) and Koester, Allard, \& Vauclair (1994) derive $T_{\text {eff }}$ near 12,400
K, while Bergeron et al. (1995), using a less efficient model for convection, derives $T_{\text {eff }}=11,600 \mathrm{~K}$.

Bradley (1996) used the mode identification and the observed periods of the three largest known pulsation modes to derive a hydrogen layer mass lower limit of $10^{-6} M_{*}$ and a best estimate of $1.5 \times 10^{-4} M_{*}$, assuming $k=2$ for the $215 \mathrm{~s}$ mode, and $20: 80 \mathrm{C} / \mathrm{O}$ core mass. The core composition is constrained mainly by the presence of the small $304 \mathrm{~s}$ pulsation.

\section{OBSERVATIONS}

We obtained $19.6 \mathrm{hr}$ of time series photometry in $1996 \mathrm{De}-$ cember and 1997 February, plus $18.8 \mathrm{hr}$ in 1999 March and December, using the three-star (Kleinman, Nather, \& Phillips 1996) photometer on the $2.1 \mathrm{~m}$ Struve telescope at McDonald Observatory.

To maximize the signal-to-noise ratio, we observed unfiltered light, since the nonradial $g$-mode light variations have the same phase in all colors (Robinson, Kepler, \& Nather 1982). G117B15A has $V=15.52$ (Eggen \& Greenstein 1965).

\section{DATA REDUCTION}

We reduce and analyze the data in the manner described by Nather et al. (1990) and Kepler (1993). We bring all the data to the same fractional amplitude scale and transform the observatories' UTC times to the uniform barycentric coordinate timescale (TCB; Standish 1998), using JPL DE96 ephemeris as our basic solar system model (Stumpff 1980). Kaspi et al. (1994) show that the effects of using different atomic timescales and ephemeris are negligible. We compute Fourier transforms for each individual run and verify that the main pulsation at 215 s dominates each data set and has a stable amplitude.

\section{TIMESCALE FOR PERIOD CHANGE}

As the dominant pulsation mode at $P=215 \mathrm{~s}$ has a stable frequency and amplitude since our first observations in 1975, we can calculate the time of maximum for each new run and look for deviations due to evolutionary cooling. 


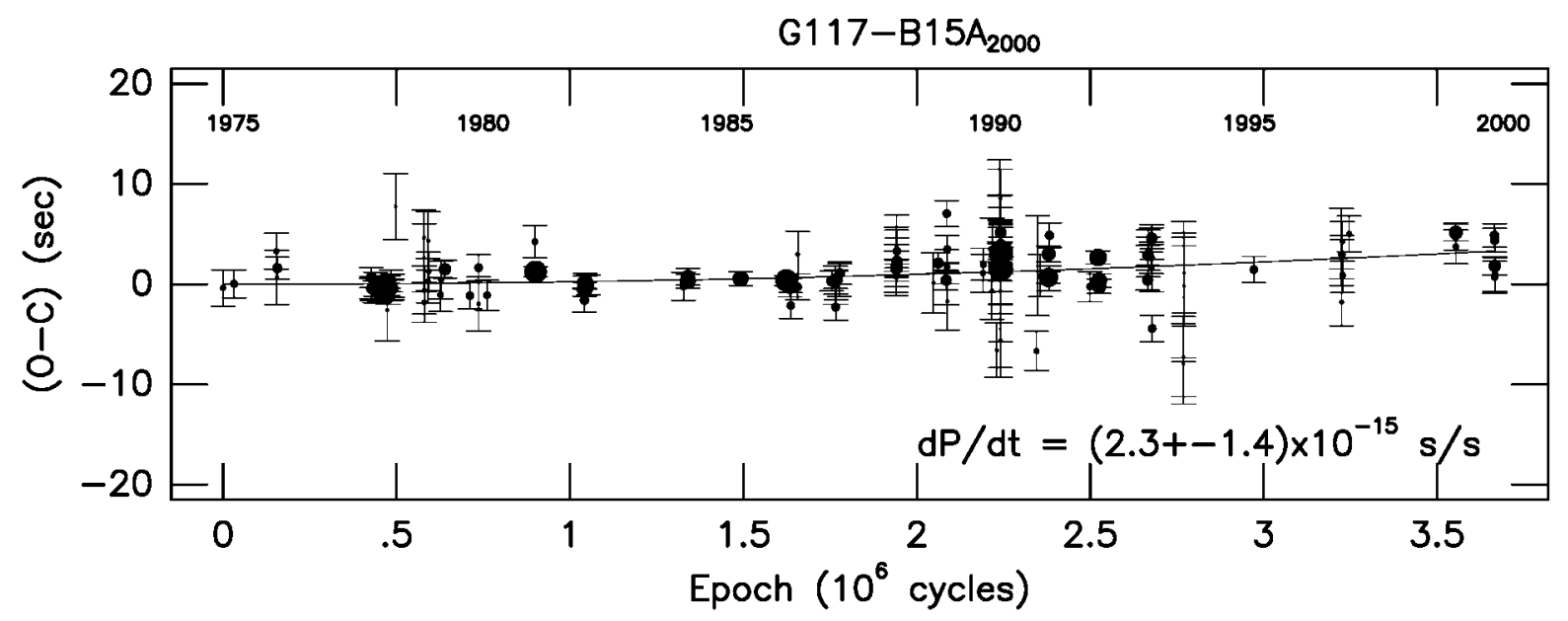

FIG. 1.- $(O-C)$ : observed minus calculated times of maxima for the $215 \mathrm{~s}$ pulsation of G117-B15A. The size of each point is proportional to its weight, i.e., inversely proportional to the uncertainty in the time of maxima squared. We show $2 \sigma$ error bars for each point, and the line shows our best-fit parabola to the data. Note that as the period of pulsation is $215.197 \mathrm{~s}$, the whole plot shows only $\pm 36^{\circ}$ in phase. At the top of the plot, we show the year of the observation.

We fit our observed time of maximum light to the equation

$$
(O-C)=\Delta E_{0}+\Delta P E+\frac{1}{2} P \dot{P} E^{2},
$$

where $\Delta E_{0}=\left(T_{\max }^{0}-T_{\max }^{1}\right), \Delta P=\left(P-P_{t=T_{\max }^{0}}\right)$, and $E$ is the epoch of the time of maximum, i.e., the number of cycles after our first observation.

In Figure 1, we show the $O-C$ timings after subtracting the correction to period and epoch and our best-fit curve through the data. From our data through 1999, we obtain a new value for the epoch of maximum, $T_{\max }^{0}=$ $2,442,397.917509 \mathrm{TCB} \pm 0.5 \mathrm{~s}$, a new value for the period, $P=215.1973907 \pm 0.0000006 \mathrm{~s}$, and most importantly, a rate of period change of

$$
\dot{P}=(2.3 \pm 1.4) \times 10^{-15} \mathrm{~s} \mathrm{~s}^{-1} .
$$

We use linear least squares to make our fit, with each point weighted inversely proportional to the uncertainty in the time of maxima for each individual run squared. We quadratically add an additional $1.8 \mathrm{~s}$ of uncertainty to the time of maxima for each night to account for external uncertainty caused perhaps by the beating of small-amplitude pulsations (Kepler et al. 1995) or small-amplitude modulation.

The estimated $\dot{P}$ is substantially different from the value estimated in 1991. The apparent reason is a scatter of the order of $1.8 \mathrm{~s}$ present in the measured times of maxima. Kepler et al. (1995) discuss the possibility of such scatter being caused by modulation due to nearby frequencies, and Costa, Kepler, \& Winget (1999) show that the real uncertainties must include the effect of all periodicities present. The 1991 value did not include such scatter in the uncertainty estimation and resulted in an overestimated statistical accuracy. We now treat this scatter as an external source of noise.

\section{CORE COMPOSITION}

For a given mass and internal temperature distribution, theoretical models show that the rate of period change increases if the mean atomic weight of the core is increased, for models that have not yet crystallized in their interiors. This applies to G117-B15A, since it is not cool enough to have a crystallized core (Winget et al. 1997). Bradley, Winget, \& Wood (1992) and Bradley (1998) compute rates of period change for models that are applicable to G117-B15A, and we summarize the relevant results here. The models of Bradley et al. (1992) and Bradley (1998) are full evolutionary models that include compositional stratification, accurate physics, and use the most recent neutrino emission rates. We refer the reader to Bradley et al. (1992) and Bradley (1996, 1998) for further details.

Two major known processes govern the rate of period change in the theoretical models of the ZZ Ceti stars: residual gravitational contraction, which causes the periods to become shorter, and cooling of the star, which increases the period as a result of the increasing degeneracy (Winget, Hansen, \& Van Horn 1983), given by

$$
\frac{d \ln P}{d t}=-a \frac{d \ln T_{c}}{d t}+b \frac{d \ln R}{d t},
$$

where $a$ and $b$ are constants associated with the rate of cooling and contraction, respectively, and are of order unity.

Following Kawaler, Winget, \& Hansen (1985), we can write

$$
\frac{d \ln P}{d t}=(-a+b s) \frac{d \ln T_{c}}{d t},
$$

where $s$ is the ratio of the contraction rate to the cooling rate

$$
s \frac{d \ln T_{c}}{d t}=\frac{d \ln R}{d t}
$$

or

$$
s=\frac{d \ln R}{d \ln T_{c}} .
$$

The $d t$ terms cancel because we evaluate the derivative as the differences in the radius, core temperature, and age between two models. Spectroscopic $\log g$ values suggest that G117- 
B15A has a mass between $0.53 M_{\odot}$ (Koester \& Allard 2000) and $0.59 M_{\odot}$ (Bergeron et al. 1995), and this agrees with the preferred seismological mass range of $0.55-0.60 M_{\odot}$ (Bradley 1998 ). For a DA white dwarf near $12,000 \mathrm{~K}$, the radius is about $9.6 \times 10^{8} \mathrm{~cm}$, with a contraction rate of about $1 \mathrm{~cm} \mathrm{yr}^{-1}$. The core temperature is about $1.2 \times 10^{7} \mathrm{~K}$, with a cooling rate of about $0.05 \mathrm{~K} \mathrm{yr}^{-1}$. With these numbers, $s$ is about 0.025 , which confirms our expectation that the rate of period change is dominated by cooling. Other processes, such as rotational spindown and magnetic fields, must be small because we do not see reliable evidence of either in the fine-structure splitting of the observed frequencies.

Bradley (1998) give a $\dot{P}$ value of $3.7 \times 10^{-15} \mathrm{~s} \mathrm{~s}^{-1}$ and find a spread of $\pm 1 \times 10^{-15} \mathrm{~s} \mathrm{~s}^{-1}$, predicted by the range of acceptable models for G117-B15A, with the $0.60 M_{\odot}$ models having the smaller values. His predicted value is within the $1 \sigma$ error bars of the observed value; a more precise observational $\dot{P}$ determination could in principle suggest a favored stellar mass.

Bradley's (1998) models are typically about $80 \%$ oxygen, and Bradley et al. (1992) describe in detail the effect of changing the core composition from pure carbon to pure oxygen for 0.50 and $0.60 M_{\odot}$ models. They also show that the predicted $\dot{P}$-value from an oxygen core model is about $15 \%-20 \%$ larger than for an equivalent carbon core model, rather than the $33 \%$ predicted by Mestel (1952) cooling theory. This reduction in $\dot{P}$ from Mestel theory is the result of the ions being a Coulomb liquid, rather than an ideal gas as assumed by Mestel theory.

The $\dot{P}$-values quoted above are for the case in which the 215 s mode is not trapped (see Bradley 1996 for details), and Bradley et al. (1992) show that if the $215 \mathrm{~s}$ mode is trapped, then the predicted $\dot{P}$ value could be as little as half the values predicted by Bradley (1998) and quoted above. In recent years, the $\dot{P}$ determinations have fluctuated between about $1 \times$ $10^{-15}$ and $3 \times 10^{-15} \mathrm{~s} \mathrm{~s}^{-1}$ (see Table 1 ), so the values predicted by seismological models are still consistent with the observations. Reducing the observational errors to about half the present value of $1.4 \times 10^{-15} \mathrm{~s} \mathrm{~s}^{-1}$ would provide enough of a constraint to confront the model predictions.

\section{REFLEX MOTION}

The presence of an orbital companion could contribute to the period change we have detected. When a star has an orbital companion, the variation of its line-of-sight position with time produces a variation in the time of arrival of the pulsation maxima by changing the light-travel time between the star and the observer. Kepler et al. (1991) calculated the possible contribution to $\dot{P}$ caused by reflex orbital motion of the observed proper-motion companion of G117-B15A as $\dot{P} \leq 1.9 \times$ $10^{-15} \mathrm{~s} \mathrm{~s}^{-1}$. If the orbit is highly eccentric and G117-B15A is near periastron, the orbital velocity could not be higher than twice that derived above, or it would exceed escape velocity. The above derivation assumed that the orbit is nearly edge-on to give the largest effect possible. Therefore, $\dot{P}_{\text {orb }} \leq 3.8 \times$ $10^{-15} \mathrm{~s} \mathrm{~s}^{-1}$.

The upper limit to the rate of period change could also be expected if a planet of Jupiter's mass were orbiting the white dwarf at a distance of $24 \mathrm{AU}$, which corresponds to an orbital period of $118 \mathrm{yr}$, or a smaller planet in a closer orbit. Note that reflex motion produces sinusoidal variations on the $O-$ $C$, which are only distinguishable from parabolic variations after a significant portion of the orbit has been covered. As we
TABLE 1

Selected $\dot{P}$ Values Derived IN
THE 1990s
\begin{tabular}{cc}
\hline Year \\
\hline $1992 \ldots \ldots$ & $(3.2 \pm 3.0) \times 10^{-15}$ \\
$1995 \ldots \ldots$ & $(1.2 \pm 2.9) \times 10^{-15}$ \\
$1997 \ldots \ldots$ & $(1.2 \pm 2.2) \times 10^{-15}$ \\
$1999 \ldots \ldots$ & $(2.8 \pm 1.7) \times 10^{-15}$ \\
$2000 \ldots \ldots$ & $(2.3 \pm 1.4) \times 10^{-15}$ \\
\hline
\end{tabular}

have observed the star for $25 \mathrm{yr}$, a sinusoid with a period shorter than $100 \mathrm{yr}$ can be discarded, but if the orbiting object were near apoastron in a highly eccentric orbit, the difference would be harder to distinguish.

\section{PROPER MOTION}

Pajdosz (1995) discusses the influence of the proper motion of the star on the measured $\dot{P}$ :

$$
\dot{P}_{\mathrm{obs}}=\dot{P}_{\mathrm{evol}}\left(1+v_{r} / c\right)+P \dot{v}_{r} / c
$$

where $v_{r}$ is the radial velocity of the star. Assuming $v_{r} / c \ll 1$, he derived

$$
\dot{P}_{\mathrm{pm}}=2.430 \times 10^{-18} \mathrm{P}[\mathrm{s}]\left(\mu\left[\operatorname{arcsec} \mathrm{yr}^{-1}\right]\right)^{2}(\pi[\operatorname{arcsec}])^{-1},
$$

where $\dot{P}_{\mathrm{pm}}$ is the effect of the proper motion on the rate of period change, $P$ is the pulsation period, $\mu$ is the proper motion, and $\pi$ is the parallax. He also calculated that for G117-B15A, $\dot{P}_{\mathrm{pm}} \simeq(8.0 \pm 0.4) \times 10^{-16} \mathrm{~s} \mathrm{~s}^{-1}$, using the proper motion $\left(\mu=0.136 \pm 0.002 \operatorname{arcsec} \mathrm{yr}^{-1}\right)$ and parallax $(\pi=0.012 \pm$ 0"005) measured by Harrington \& Dahn (1980). With the parallax by Van Altena, Lee, \& Hoffleit (1995) of $\pi=$ $0.0105 \pm 0$ ".004 and the above proper motion, we calculate $\dot{P}_{\mathrm{pm}}=(9.2 \pm 0.5) \times 10^{-16} \mathrm{~s} \mathrm{~s}^{-1}$.

The upper limit to the observed $\dot{P}$ is already only a few times the $\dot{P}$ expected from proper motion alone.

\section{CONCLUSIONS}

While it is true that the period change timescale can be proportional to the cooling timescale, other phenomena with shorter timescales can affect $\dot{P}$. The cooling timescale is the longest possible one. As a corollary, if the observed $\dot{P}$ is low enough to be consistent with evolution, then other processes (such as perhaps a magnetic field) are not present at a level sufficient to affect $\dot{P}$.

We compare the observed value of $\dot{P}$ with the range of theoretical values derived from realistic evolutionary models with carbon/oxygen cores subject to $g$-mode pulsations in the temperature range of G117-B15A. The adiabatic pulsation calculations of Bradley (1996), and Brassard et al. (1992, 1993), which allow for mode trapping, give $\dot{P} \simeq(2-7) \times 10^{-15} \mathrm{~s} \mathrm{~s}^{-1}$ for the $l=1$, low- $k$ oscillation observed. The observed $3 \sigma$ upper limit, $\dot{P} \leq 6.5 \times 10^{-15} \mathrm{~s} \mathrm{~s}^{-1}$, corresponding to a timescale for period change of $P / \dot{P} \leq 1.2 \times 10^{9} \mathrm{yr}$, equivalent to $1 \mathrm{~s}$ in $6 \times 10^{6} \mathrm{yr}$, is within the theoretical predictions and very close to it.

Our upper limit to the rate of period change brings us to realms in which reflex motion from the proper-motion companion, if they form a physical binary, or an unseen orbiting 
planet is of the same order as the evolutionary timescale. The effect of proper motion of the star itself is only a few times smaller. These two effects must therefore be accurately measured. We are on the way to measure the evolutionary timescale for this lukewarm white dwarf, but the observed phase scatter of the order of $1.8 \mathrm{~s}$ increased the baseline necessary for a measurement. This scatter is still present in our measurement.

This work was partially supported by grants from $\mathrm{CNPq}$ (Brazil), FINEP (Brazil), NSF (USA), and NASA (USA).

\section{REFERENCES}

Bergeron, P., Wesemael, F., Lamontagne, R., Fontaine, G., Saffer, R. A., \& Allard, N. F. 1995, ApJ, 449, 258

Bradley, P. A. 1996, ApJ, 468, 350

1998, ApJS, 116, 307

Bradley, P. A., Winget, D. E., \& Wood, M. A. 1992, ApJ, 391, L33

Brassard, P., Fontaine, G., Wesemael, F., \& Talon, A. 1993, in White Dwarfs: Advances in Observation and Theory, ed. M. A. Barstow (Dordrecht: Kluwer), 485

Brassard, P., Fontaine, G., Wesemael, F., \& Tassoul, M. 1992, ApJS, 81, 747

Costa, J. E. S., Kepler, S. O., \& Winget, D. E. 1999, ApJ, 522, 973

Eggen, O. J., \& Greenstein, J. L. 1965, ApJ, 141, 83

Harrington, R. S., \& Dahn, C. C. 1980, AJ, 85, 454

Kaspi, V. M., Taylor, J. H., \& Ryba, M. F. 1994, ApJ, 428, 713

Kawaler, S. D., Winget, D. E., \& Hansen, C. J. 1985, ApJ, 295, 547

Kepler, S. O. 1984, ApJ, 286, 314 . 1993, Baltic Astron., 2, 515

Kepler, S. O., Robinson, E. L., Nather, R. E., \& McGraw, J. T. 1982, ApJ, 254, 676

Kepler, S. O., et al. 1991, ApJ, 378, L45 1995, Baltic Astron., 4, 221

Kleinman, S. J., Nather R. E., \& Phillips, T. 1996, PASP, 108, 356

Koester, D., \& Allard, N. F. 2000, Baltic Astron., in press
Koester, D., Allard, N. F., \& Vauclair, G. 1994, A\&A, 291, L9

McGraw, J. T. 1979, ApJ, 229, 203

McGraw, J. T., \& Robinson, E. L. 1976, ApJ, 205, L155

Mestel, L. 1952, MNRAS, 112, 583

Nather, R. E., Winget, D. E., Clemens, J. C., Hansen, C. J., \& Hine, B. P. 1990, ApJ, 361, 309

Pajdosz, G. 1995, A\&A, 295, L17

Robinson, E. L. 1979, in IAU Colloq. 53, White Dwarfs and Variable Degenerate Stars, ed. H. M. Van Horn \& V. Weidemann (Rochester: Univ. Rochester), 343

Robinson, E. L., Kepler, S. O., \& Nather, R. E. 1982, ApJ, 259, 219

Robinson, E. L., et al. 1995, ApJ, 438, 908

Standish, E. M. 1998, A\&A, 336, 381

Stumpff, P. 1980, A\&AS, 41, 1

van Altena, W. F., Lee, J. T., \& Hoffleit, E. D. 1995, The General Catalogue of Trigonometric Parallaxes (4th ed.; New Haven: Yale Univ. Obs.)

Winget, D. E., Hansen, C. J., \& Van Horn, H. M. 1983, Nature, 303, 781

Winget, D. E., Kepler, S. O., Kanaan, A., Montgomery, M. H., \& Giovannini, O. 1997, ApJ, 487, L191

Wood, M. A., \& Winget, D. E. 1988, in Multimode Stellar Pulsations, ed. G. Kovacz, L. Szabados, \& B. Szeidl (Budapest: Konkoly Obs.-Kultura), 199 